\title{
Tuberculosis of the Knee: A Case Report and Literature Review
}

\author{
Francesco M. Uboldi ${ }^{1}$ Silvia Limonta ${ }^{2}$ Paolo Ferrua ${ }^{1} \quad$ Andrea Manunta $^{3}$ Antonio Pellegrini ${ }^{4}$
}

1 Department of Knee Surgery, Gaetano Pini Hospital, Milan, Italy

2 rd Division of Infectious Diseases, University of Milan, Luigi Sacco Hospital, Milan, Italy

3 Orthopedic Unit, University of Sassari, Sassari, Italy

4 Reparative Orthopedic Surgery and Infectious Complications Unit, San Siro Clinical Institute, Galeazzi Orthopedic Institute, Milan, Italy

Joints 2017;5:180-183.

\author{
Address for correspondence Francesco M. Uboldi, MD, Department \\ of Knee Surgery, Gaetano Pini Hospital, Piazza Cardinal Ferrari 1 , \\ 20122 Milan, Italy (e-mail: fraubo@gmail.com).
}

\author{
Abstract \\ Keywords \\ - skeletal \\ - tuberculosis \\ - knee \\ - arthritis \\ - therapy
}

Tuberculosis (TB) is currently in resurgence due to immigration from endemic areas. Skeletal TB frequently mimics more common etiologies and can be difficult to diagnose. A case of TB knee arthritis in a young woman with painful and swelling knee is reported here. Arthrotomy was performed and inflamed synovial tissue was found, with multiple rice bodies in the eroded lateral femoral condyle. The patient was treated with an antituberculosis polytherapy and at 1-year follow-up, she reported relief from pain and swelling. We believe that all surgeons assessing patients from TB endemic regions have to adopt an updated approach to TB treatment. Thus, a literature review is also reported here on the current strategies used in different knee TB cases.

\section{Introduction}

Although tuberculosis (TB) is not a common disease in industrialized countries, it remains a common and endemic illness in developing countries. ${ }^{1}$ Every year, approximately 10.4 million infected people develop active disease, resulting in almost 1.4 million deaths annually. ${ }^{2}$

Although the majority of newly diagnosed cases have pulmonary $\mathrm{TB}$, a considerable number of organs, bones, and joints can be affected too. In particular, skeletal TB accounts for approximately 10 to $35 \%$ of extrapulmonary cases, ${ }^{3}$ and the knee is the third most frequently affected site after spine and hip. ${ }^{4}$ In Italy, TB incidence is 10 cases per 100,000 inhabitants. ${ }^{5}$ According to this data, active pulmonary TB was simultaneously detected only in one third of patients suffering from skeletal TB. Osteoarticular TB remained a diagnostic challenge, because in the majority of cases, the disease is detected late when lesions are paucibacillary and smears are in most cases not diriment. ${ }^{6,7}$

The aim of this review was to report a recent clinical case of TB of knee to highlight that a high level of suspicion might be adopted to establish the correct diagnosis of joint TB. Besides, an update on current concepts of clinical and surgery therapies is given.

\section{Case Presentation}

A 29-year-old Philippine woman suffering from knee pain was referred to our Septic Orthopaedic Care Unit in 2015. We obtained patient consent to publish her clinical case data.

According to her history, she complained of continuous and progressive lateral knee pain since 1 year that forced her to use two crutches to walk. No constitutional symptoms were reported. She had visited her native country few months before symptoms appeared and reported that one of her brothers and her father had just been treated for pulmonary TB. At physical examination, her knee was swollen and loss of motion was observed (range of movement 0-100 degrees). Pressure pain could be induced on lateral femoral condyle (LFC). Liquid collected from knee arthrocentesis was clear. Blood investigations revealed elevated C-reactive protein (CRP) of $5.4 \mathrm{mg} / \mathrm{dL}$, erythrocyte sedimentation rate (ESR) of $35 \mathrm{~mm} / \mathrm{h}$, and white blood cell count of $9.19 \times 10^{9} / \mathrm{L}$. Chest X-ray showed obscured costodiaphragmatic recesses and signs of a previous pleurisy. Knee X-ray showed a bone lesion in the LFC ( - Fig. 1). Magnetic resonance showed a $1.5 \times 1.5 \mathrm{~cm}$ focal lesion with subcortical development, hypointense in T1-weighted sequences, and with hypointense core plus hyperintense surrounding in
Copyright (c) 2017 Georg Thiem

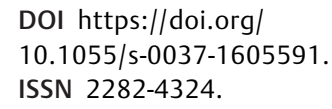
$10.1055 / \mathrm{s}-0037-1605591$. ISSN 2282-4324.

DOI https://doi.org/

Verlag KG Stuttgart · New York published online

August 11, 2017 (c) (i) 


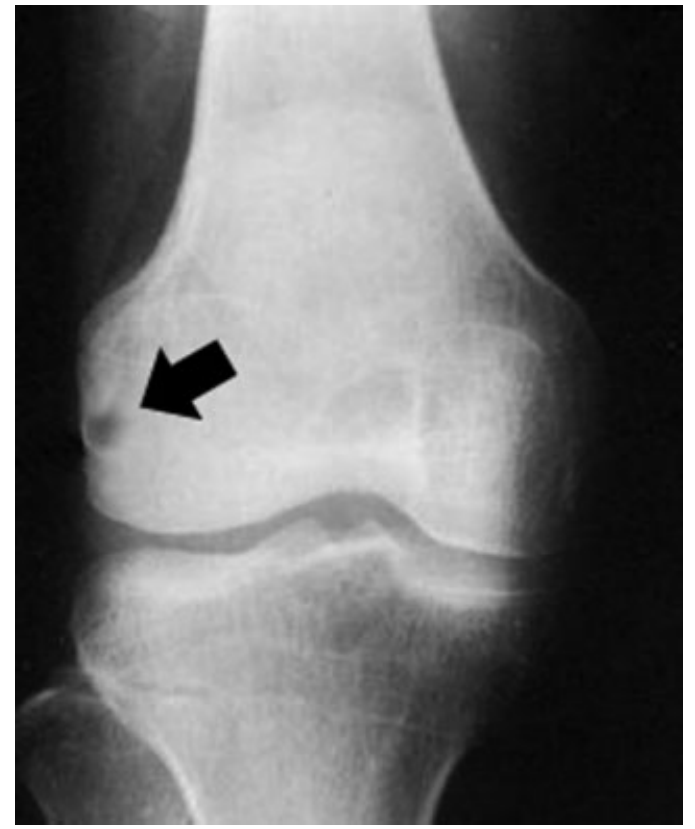

Fig. 1 X-ray of the knee showing a bone lesion (arrow) of lateral femoral condyle.

T2-weighted sequences (-Fig. 2). Computed tomography (CT) scans confirmed the lesion, showing a cortical bone interruption surrounded by an area of osteolysis in the LFC.

The patient underwent a surgical LFC curettage and synovectomy with synovial biopsy that was found to be inflamed and hypertrophied with multiple "rice bodies" (-Fig. 3). The histopathologic exam revealed granulomatous inflammation with caseous necrosis, suggestive of TB. Polymerase chain reaction (PCR) test for Mycobacterium tuberculosis DNA was positive, resulting in an isoniazid monoresistance profile. The patient was treated with a polytherapy consisting of rifampin, pyrazinamide, and

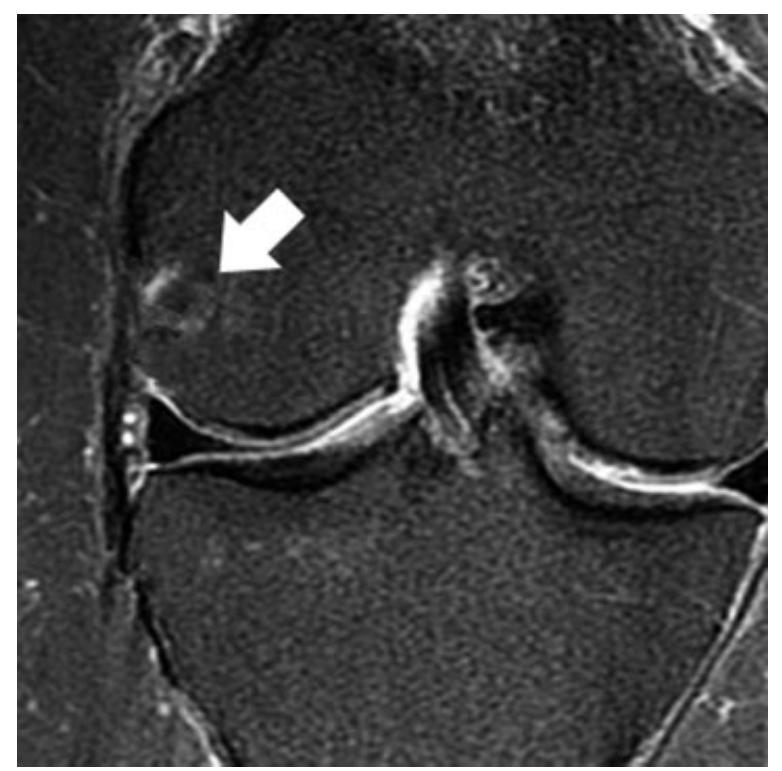

Fig. 2 T2-weighted MR scan with hypointense core plus hyperintense surrounding the lesion (arrow). MR, magnetic resonance.

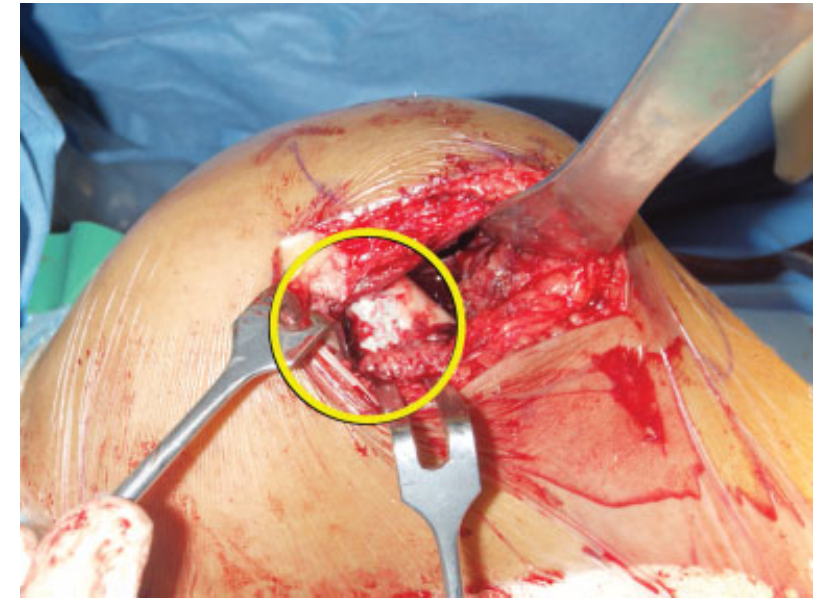

Fig. 3 Rice bodies in the lesion of lateral femoral condyle.

ethambutol that continued for 1 year. At 2-year follow-up, the patient reported relief from pain and swelling with knee movements from 0 to 120 degrees.

\section{Discussion}

In low-incidence European countries, a substantial proportion of TB patients comes from developing countries, where the disease is more common among young adults. In our reported case, the patient was a 29-year-old Philippine woman.

Identification of $M$. tuberculosis is essential for definitive diagnosis of TB arthritis based on culture or histopathologic examinations. Acid-fast stain test results positive in only 0 to $5 \%$ of cases, while $M$. tuberculosis is isolated in approximately 60 to $90 \%$ of cases. ${ }^{8}$

Medical treatment of TB is necessarily based on a combination therapy, divided into two phases: the initial phase (usually based on four drugs) lasts 2 months, and the continuation phase (usually with two drugs administered) can last from 4 to 7 months or more in specific cases. ${ }^{9,10}$ The optimal duration of therapy in musculoskeletal TB is uncertain. For most patients, 6 to 9 months of treatment is enough, ${ }^{11}$ but a longer duration (9-12 months) is warranted for patients with extensive or advanced disease. ${ }^{12}$ The approach to treatment of monoresistant TB depends on the drug to which the isolate is resistant. Isoniazid monoresistance is the most common type as in our case report. These patients are usually treated with rifampin, pyrazinamide, and ethambutol for 6 to 9 months, with no difference between initial and continuation phase. ${ }^{13,14}$ Effective therapy for isoniazid monoresistant TB is associated with very high bacteriologic and clinical response rates (>95\%) and low relapse rates $(<5 \%){ }^{13}$

When knee TB is limited to bones, the most common treatment in adult patients is arthrotomy and curettage of the lesion. Complete debridement is crucial for an effective treatment of TB arthritis, which can eradicate infected tissues to lower risks of disease reactivation. According to Vohra et al, $^{15}$ CT scan and MR are helpful in accurately 
localizing skeletal lesions resulting from TB, while surgical resection of the pathological mass can completely eliminate the symptoms. ${ }^{16}$ The prognosis is better in articular disease, as there is less deformity as well as less residual pain and disability. ${ }^{17}$

Old surgical options consisted of articular debridement and joint fusion as the best treatment, but postoperative joint function was poor and had a serious impact on patients' working and daily living activities. Moreover, a 1 to $9 \%$ of recurrence rate has been reported. Unfortunately, sometimes joint fusion is the only viable treatment option. Arthrodesis may be more appropriate for patients with minimal bone loss and broad cancellous surface, which allows a good bone apposition and compression.

Patients with end-stage TB arthritis, severe damage to joints, and periarticular abscess who undergo a single debridement may have a relatively higher recurrence rate. ${ }^{18}$ Teo et $\mathrm{al}^{19}$ reported on an isolated case of highly erosive knee TB in a 35-year-old fit Burmese worker who had migrated to Malaysia. The patient was first treated with arthrotomy, debridement, and full-length back slab immobilization, but the treatment was unsuccessful and 5 months later, a knee arthrodesis was performed using an Ilizarov frame. ${ }^{19}$ Open debridement and arthrodesis are the main treatment options for this condition in adults. Several techniques have been described for knee fusion, including internal fixation with single plate, double plate, antegrade locking nail, modular intramedullary nails, and external fixation. Tang et $\mathrm{al}^{20}$ reported on 26 patients who underwent open debridement and fusion with a unilateral external fixator (EF) combined with crossed cannulated screws, intended to increase stiffness while limiting the size of the EF, thereby minimizing the risk of TB dissemination. Complete fusion was achieved within 8 months, with a mean postoperative alignment of 5.4 degrees valgus and 12.5 degrees flexion, and a mean leg length discrepancy of $2.8 \mathrm{~cm} .^{20}$

Total knee arthroplasty (TKA) can help patients to get relief from pain and restoration of a good knee function. However, a stationary knee TB is required for this kind of treatment. Attempt to eradicate TB should be made before implanting a TKA. Habaxi et $\mathrm{al}^{21}$ suggested prolonging antiTB treatment until there was a downward trend in ESR levels, possibly under $40 \mathrm{~mm} / \mathrm{h}$. Furthermore, surgeons should make sure to clean out the joint capsule and synovial tissue necrosis, as well as to check for sinuses.

Habaxi et $\mathrm{al}^{21}$ reported on 10 patients where no dislocations or aseptic loosening of TKA was reported with an average HSS score of $86.7 \pm 5.4$ and ROM improvement to $95 \pm 5$ degrees at follow-up; only one patient had recurrence and a two-stage revision operation was performed.

Some authors believe that cemented TKA should be preferred in knee TB to fill the possible bone defects. Moreover, the production of heat during bone cement polymerization can kill Mycobacterium and lower the recurrence rate. ${ }^{21}$

Klein and Jacquette ${ }^{22}$ reported a case of TB arthritis of the knee in a 36-year-old Haitian woman treated with TKA that failed due to a misinterpreted diagnosis. A static antibioticimpregnated cement spacer after removal of prosthetic components was used, and a new prosthesis was implanted after 7 months of four-drug regimen, added with a daily oral moxifloxacin and thrice weekly intravenous amikacin for sterilization of joint space.

TB arthritis after TKA is clinically indistinguishable from infections caused by most pathogenic bacteria. Although it is a rare presentation, it should be easily detected, especially in patients with predisposing conditions with a history of TB infection. Indeed, late diagnosis of TB after TKA requires implant removal. However, when patients are promptly treated, joint retention is possible and could have long viability.

In a suspected TB infection after TKA, empiric anti-TB treatment should be administered based on clinical diagnosis, and since a viable pathogen is required for susceptibility testing, alternative culture material might be used (wound drainage, joint aspiration, lung or genitourinary tract samples). ${ }^{22}$ The evolution of the treatment is often favorable. Lara-Oya et $\mathrm{al}^{23}$ published a literature review on 17 cases reporting a disease onset between 2 months and 15 years (45 months average) after TKA with only one failed case.

TB of the patellar bone is rare with an incidence of 0.09 to $0.15 \%{ }^{24}$ MacLean et $\mathrm{al}^{25}$ published a case of TB of the patella mimicking prepatellar bursitis. The first diagnosis was based on clinical appearance and on a Staphylococcus aureus positive test of fluid collected from bursitis. Successively, X-ray showed a central nidus of necrotic bone in the patella and MRI confirmed osteomyelitis. Treatment options depend on the extent and spread of osteomyelitis. Curettage may be used to remove the sequestrum and establish a tissue diagnosis. Prepatellar bursitis is frequent, but in high-risk patients, further diagnostic tests and imaging studies are required to facilitate diagnosis. ${ }^{17,25,26}$

\section{Conclusion}

The greatest challenge in diagnosing osteoarticular TB is to take diagnosis of the disease into consideration, especially in nonendemic countries where TB is infrequent. A high level of suspicion for TB should be recommended in every infection of knee joint, especially after TKA.

\section{References}

1 Amini J, Poka H, Kumbu J, et al. The crisis of tuberculosis in Papua New Guinea-the role of older strategies for public health disease control. P N G Med J 2012;55(01):(04):1-4

2 Global Tuberculosis Report 2016, WHO. Available at: http://www. who.int/entity/tb/publications/global_report/gtbr2016_main_text.pdf?ua=1. Accessed June 12, 2017

3 Peto HM, Pratt RH, Harrington TA, LoBue PA, Armstrong LR. Epidemiology of extrapulmonary tuberculosis in the United States, 1993-2006. Clin Infect Dis 2009;49(09):1350-1357

4 Tuli SM. Tuberculosis of the Skeletal System. 4th ed. New Delhi: Jaypee Brothers; 2010

5 La tubercolosi in Italia Rapporto 2008, Istituto Superiore di Sanità. Available at: http://www.salute.gov.it/imgs/C_17_pubblicazioni_1472_allegato.pdf. Accessed June 12, 2017 
6 Mkandawire NC, Kaunda E. Bone and joint TB at Queen Elizabeth Central Hospital 1986 to 2002. Trop Doct 2005;35(01):14-16

7 Raviglione MC, Narain JP, Kochi A. HIV-associated tuberculosis in developing countries: clinical features, diagnosis, and treatment. Bull World Health Organ 1992;70(04):515-526

8 Hopewell PC. Overview of Clinical Tuberculosis. In: Bloom BR, ed. Tuberculosis: pathogenesis, protection, and control. Washington, DC: American Society for Microbiology; 2005:25-46

9 Blumberg HM, Burman WJ, Chaisson RE, et al; American Thoracic Society, Centers for Disease Control and Prevention and the Infectious Diseases Society. American Thoracic Society/Centers for Disease Control and Prevention/Infectious Diseases Society of America: treatment of tuberculosis. Am J Respir Crit Care Med 2003;167(04):603-662

10 Hopewell PC, Pai M, Maher D, Uplekar M, Raviglione MC. International standards for tuberculosis care. Lancet Infect Dis 2006;6 (11):710-725

11 American Thoracic Society. Treatment of tuberculosis. MMWR Recomm Rep 2003;52(RR-11):1-77

12 Blumberg HM, Leonard MK Jr, Jasmer RM. Update on the treatment of tuberculosis and latent tuberculosis infection. JAMA 2005;293(22):2776-2784

13 Cattamanchi A, Dantes RB, Metcalfe JZ, et al. Clinical characteristics and treatment outcomes of patients with isoniazidmonoresistant tuberculosis. Clin Infect Dis 2009;48(02):179-185

14 Centers for Disease Control and Prevention. Prevention and treatment of tuberculosis among patients infected with human immunodeficiency virus: principles of therapy and revised recommendations. MMWR Recomm Rep 1998;47(RR-20):1-58

15 Vohra R, Kang HS, Dogra S, Saggar RR, Sharma R. Tuberculous osteomyelitis. J Bone Joint Surg Br 1997;79(04):562-566
16 Elmi A, Tabrizi A, Tolouei FM. Skeletal tuberculosis presenting as a small cystic lesion in the medial femoral condyle. Arch Bone Jt Surg 2013;1(02):112-115

17 Dhillon MS, Aggarwal S, Prabhakar S, Bachhal V. Tuberculosis of the foot: an osteolytic variety. Indian J Orthop 2012;46(02):206-211

18 Oztürkmen Y, Uzümcügil O, Karamehmetoğlu M, Leblebici C, Caniklioğlu M. Total knee arthroplasty for the management of joint destruction in tuberculous arthritis. Knee Surg Sports Traumatol Arthrosc 2014;22(05):1076-1083

19 Teo Sh, Teh K, Azura L, Ng Y. The great mimic again? A case of tuberculosis knee. Malays Orthop J 2011;5(03):32-34

20 Tang X, Zhu J, Li Q, Chen G, Fu W, Li J. Knee arthrodesis using a unilateral external fixator combined with crossed cannulated screws for the treatment of end-stage tuberculosis of the knee. BMC Musculoskelet Disord 2015;16:197

21 Habaxi KK, Wang L, Miao XG, et al. Total knee arthroplasty treatment of active tuberculosis of the knee: a review of 10 cases. Eur Rev Med Pharmacol Sci 2014;18(23):3587-3592

22 Klein GR, Jacquette GM. Prosthetic knee infection in the young immigrant patient-do not forget tuberculosis!. J Arthroplasty 2012;27(07):1414.e1-1414.e4

23 Lara-Oya A, Lìebana-Martos M, Rodríguez-Granger J, et al. Artritis tuberculosa sobre prótesis de rodilla: descripción de un caso y revisión de la literatura. Revista Española de Quimioterapia Advance Access 2016;29(04):214-219

24 Mittal R, Trikha V, Rastogi S. Tuberculosis of patella. Knee 2006;13 (01):54-56

25 MacLean S, Kulkarni S. Tuberculosis of the patella masquerading as prepatellar bursitis. Ann R Coll Surg Engl 2013;95(01):e17-e19

26 Ciaschini M, Sundaram M. Radiologic case study. Prepatellar Morel-Lavallée lesion. Orthopedics 2008;31(07):626, 719-721 